\title{
The Morphological Basis for Binocular and ON/OFF Convergence in Tree Shrew Striate Cortex
}

\author{
E. Christopher Muly and David Fitzpatrick \\ Department of Neurobiology, Duke University Medical Center, Durham, North Carolina 27710
}

\begin{abstract}
We used retrograde and anterograde transport methods and single-cell reconstructions to examine the projection from layer IV to supragranular layers in the tree shrew's striate cortex. We found that neurons in the ON and OFF subdivisions of layer IV (IVa and IVb, respectively) have overlapping terminal fields throughout layers II and III. Despite their overlap, these projections are organized in a highly stratified, mirror-symmetric fashion that respects the vertical position of neurons within each sublayer. Neurons in the middle of layer IV (lower IVa and upper IVb) project to layers IIIa/b, II, and I; neurons located at the edges of layer IV (upper IVa and lower IVb) project to the lower half of layer IIIc; and neurons in the middle of IVa and the middle of IVb project to upper IIIc.

The stratified nature of the projections from layer IV to layer III is reminiscent of the pattern of ipsilateral and contralateral eye inputs to layer IV. Inputs from the ipsilateral eye are limited to the edges of layer IV (upper IVa and lower IVb), while those from the contralateral eye terminate throughout the depth of IVa and IVb. Thus, cells near the edges of layer IV should receive strong input from both eyes, while those in the middle of layer IV should receive mostly contralateral input. Taken together, these results suggest that the projections from layer IV to layer III bring together the information conveyed by the ON and OFF pathways, but do so in a way that matches the ocular dominance characteristics for each pathway.
\end{abstract}

One of the important functions of striate cortex is to bring about the convergence of information conveyed by different populations of lateral geniculate neurons. Perhaps the most familiar example of convergence is found in the binocular responses of cortical neurons. Most cortical neurons will respond to stimulation of either eye, whereas LGN neurons respond selectively to stimulation of the contralateral or ipsilateral eye, but not both (Hubel and Wiesel, 1961, 1962). Another form of convergence is revealed by comparing the responses of LGN and cortical neurons to the onset and offset of light in their receptive field centers. Individual neurons in the LGN respond selectively to either the onset of light (ON response) or the offset (OFF re-

Received Aug. 13, 1991; revised Nov. 1, 1991; accepted Nov. 7, 1991.

Thanks to Martha Foster for expert technical assistance and Drs. Michael Conley and Edward Callaway for helpful comments on the manuscript. This work was supported by NIH Grants EYO6821 and EYO6661.

Correspondence should be addressed to David Fitzpatrick, Department of Neurobiology, Box 3209, Duke University Medical Center, Durham, NC 27710.

Copyright (C) 1992 Society for Neuroscience $0270-6474 / 92 / 121319-16 \$ 05.00 / 0$ sponse), reflecting distinct inputs from corresponding populations of retinal ganglion cells (Kuffler, 1953; Hubel and Wiesel, 1961). In contrast, single cortical neurons have both $O N$ and OFF responses due to the convergence of information conveyed by ON and OFF center LGN neurons (Hubel and Wiesel, 1962; Schiller, 1982; Tanaka, 1983; Sherk and Horton, 1984).

The generation of binocular, dual-sign (ON/OFF) responses from monocular, single-sign (ON or OFF) responses must reflect a simple anatomical fact: the axons of four different classes of LGN neurons (i.e., ipsilateral ON, ipsilateral OFF, contralateral $\mathrm{ON}$, and contralateral OFF), or cortical neurons that convey the information from these LGN neurons, synapse on a single postsynaptic neuron. But this statement, by itself, fails to convey the variety of outcomes that exist for each form of convergence and provides little insight into how the two forms of convergence are interrelated. For example, as judged by cortical receptive field characteristics, ON and OFF inputs can be brought together in a variety of different spatial configurations. In some cortical cells (simple cells), ON and OFF response zones are separate so that stimulation of selected regions of the receptive field yields either an ON or OFF response; this class of cell can be further subdivided depending on the number and arrangement of $O N$ or OFF zones. In other cells (complex), ON and OFF response zones overlap completely so that either response can be elicited throughout the field (Hubel and Wiesel, 1962, 1968; Schiller et al., 1976; Palmer and Davis, 1981). The degree of binocular convergence also varies considerably from cell to cell. Some cells respond to both eyes but are dominated by inputs from the left eye, some are dominated by inputs from the right eye, and others have responses that fall in between these extremes (Hubel and Wiesel, 1962, 1977).

The intricate relationship between ON/OFF and binocular convergence is also revealed in the receptive field properties of cortical neurons and can be summarized as follows. First, whatever spatial distribution of ON and OFF zones is present in the receptive field of the left eye, the same distribution is found in the right; that is, the spatial arrangements of the $O N$ and OFF subfields in the two eyes are always the same (Hubel and Wiesel, 1962; Skottun and Freeman, 1984; Ohzawa and Freeman, $1986 a, b)$. Second, the relative strength of input from the two eyes (ocular dominance or preference) is the same for both signs. If the $\mathrm{ON}$ response in the left eye is twice that of the right eye, then the OFF response in the left eye will be twice that of the right eye (Hubel and Wiesel, 1962, 1977).

In order to gain some understanding of the complex neural circuitry that is responsible for bringing together the $\mathrm{ON}$ and OFF pathways from the two eyes with such variety and precision, we have been studying the input-output organization of layer IV in the tree shrew striate cortex (Fitzpatrick and Racz- 


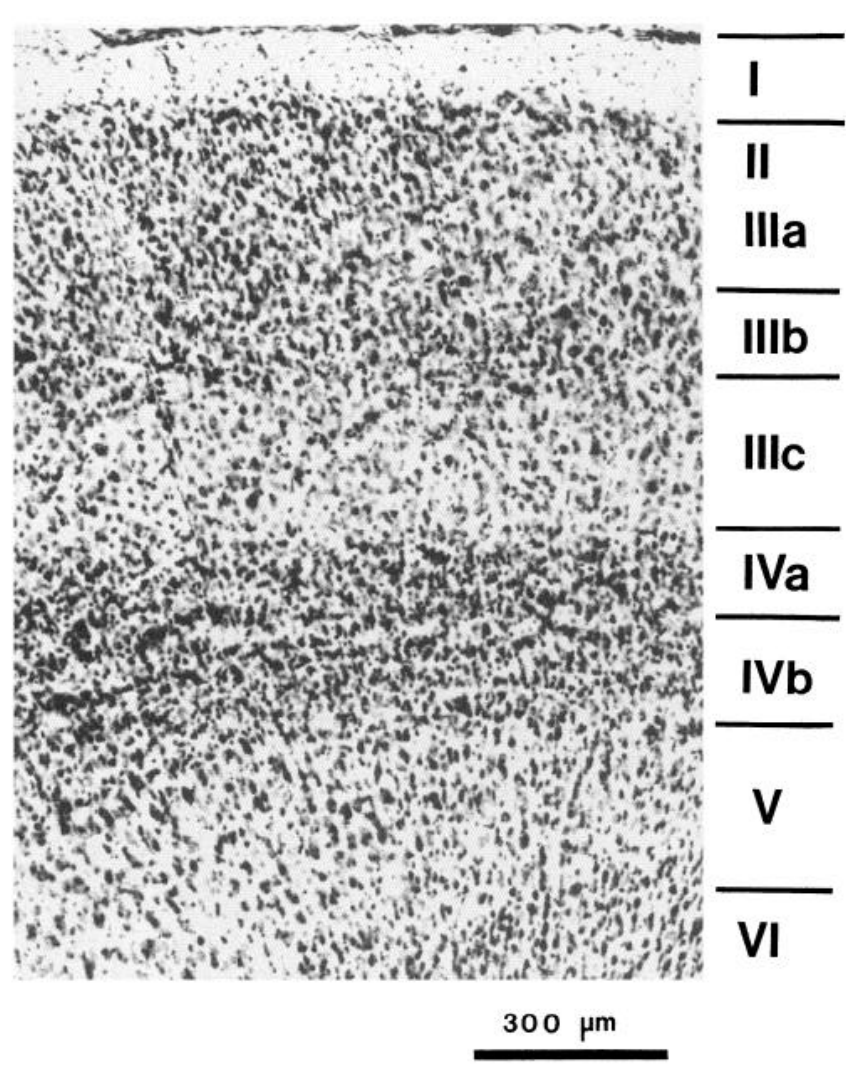

Figure 1. Photomicrograph of Nissl-stained section through tree shrew striate cortex.

kowski, 1990; Raczkowski and Fitzpatrick, 1990). In this species, ON and OFF center axons from the LGN terminate in separate horizontal tiers of layer IV (IVa and IVb, respectively) (Harting et al., 1973; Conley et al., 1984; Raczkowski and Fitzpatrick, 1990). Within each tier, there is partial segregation of input from the two eyes (Harting et al., 1973; Casagrande and Harting, 1975; Hubel, 1975; Conley et al., 1984; Raczkowski and Fitzpatrick, 1990). Axons driven by the ipsilateral eye terminate in the upper half of IVa and lower half of IVb, while axons driven by the contralateral eye terminate throughout IVa and IVb. The dendrites of the layer IV neurons contacted by these axons are also highly stratified (Geisert and Guillery, 1979), and this organization probably accounts for the fact that most neurons in IVa have ON responses, while most neurons in IVb have OFF responses (Norton et al., 1985; Kretz et al., 1986). Taken together, these results raise the possibility that ON/OFF and binocular convergence occur in a two stage process: in the first stage, the ON pathways from the two eyes are combined in layer IVa and the OFF pathways from the two eyes are combined in layer IVb; in the second stage, binocular ON and OFF pathways are brought together via the projections from layer IV to the superficial layers.

The present study tests this idea by examining the pattern of projections from layer IV to the superficial layers of striate cortex. The results will show that neurons in IVa and IVb have overlapping terminal fields throughout layers II and III. In addition, these projections are highly stratified in a way that respects the vertical position of the neurons within each subtier of layer IV. We consider the significance of these findings for understanding cortical mechanisms of ON/OFF and binocular convergence.

Some of these results have been presented in abstract form (Muly et al., 1989; Muly and Fitzpatrick, 1991).

\section{Materials and Methods}

Injection of tracers. For this study, 30 adult tree shrews, Tupaia belangeri, of both sexes and weighing 120-300 gm were used. Surgical anesthesia was induced with a mixture of ketamine/xylazine $(180 \mathrm{mg} / \mathrm{kg}$. $5.75 \mathrm{mg} / \mathrm{kg}$, i.m.) and maintained with supplements of sodium pentobarbital $(0.1-0.2 \mathrm{ml}$ of $10 \mathrm{mg} / \mathrm{ml}$, i.p. $)$.

After the animal's head was placed in a stereotaxic frame, a craniotomy in the form of a trench (approximately $5 \mathrm{~mm} \times 1 \mathrm{~mm}$ ) was performed over the occipital cortex, which was then bathed in warm saline. Micropipettes containing a mixture of horseradish peroxidase (HRP) or biocytin were lowered to various depths in the striate cortex. The HRP mixture used was 10\% HRP (Boehringer-Mannheim), $1 \%$ HRP conjugated to wheat germ agglutinin (WGA-HRP; Sigma), and $0.2 \%$ polyornithine (Sigma, $200 \mathrm{kDa}$ ) dissolved in $200 \mathrm{mM} \mathrm{KCl}, 50 \mathrm{~mm}$ Tris buffered at $\mathrm{pH}$ 7.6. The biocytin pipettes contained $5 \%$ biocytin (Sigma) in normal saline.

For HRP experiments, three to six iontophoretic injections were made in each hemisphere through tip sizes of $1-7 \mu \mathrm{m}$ using $0.2-0.5 \mu \mathrm{A}$ of positive current for 7-15 min. The small size and the separation of the injections ensured that labeled fibers or cells from different injection sites did not overlap.

For biocytin experiments, 4-10 penetrations, with one or two iontophoretic injections each, were made in area 17 in each hemisphere. If two injections were made along a given penetration, they were spaced not less than $200-300 \mu \mathrm{m}$ apart. Injections were made through tip sizes of 5-15 $\mu \mathrm{m}$ using 2.5-3.5 $\mu \mathrm{A}$ of positive continuous or pulsed current for 2-3 min each. Following the injections, the scalp was sutured and the animal returned to its cage.

Tissue processing. After a 2-3 d survival, the animal was given a lethal dose of Nembutal and perfused transcardially. The animals were perfused with phosphate-buffered saline (PBS) and then with fixative containing $3.5 \%$ paraformaldehyde and $0.5 \%$ glutaraldehyde in $0.1 \mathrm{M}$ phosphate buffer, $\mathrm{pH} 7.4(\mathrm{~PB})$, for the HRP experiments, or $4 \%$ paraformaldehyde in PB for the biocytin experiments. After $30 \mathrm{~min}$, the fixative was flushed with a solution of $10 \%$ sucrose and $2 \%$ dimethyl sulfoxide in PB, and the brain was removed, placed in $25 \%$ sucrose for $12-24 \mathrm{hr}$, and then sectioned at $50 \mu \mathrm{m}$ in the coronal plane. Sections from HRP-injected brains were processed for peroxidase histochemistry using 3,3'-diaminobenzidine (DAB) intensified with nickel and cobalt (Adams, 1981). Sections from biocytin-injected brains were incubated for $2 \mathrm{hr}$ in avidin biotinylated peroxidase complex (Vector, Elite kit) in $0.75 \%$ Triton X-100. The sections were rinsed in PBS and then placed in $0.1 \%$ glutaraldehyde for $4 \mathrm{~min}$, rinsed in PBS again, and reacted with $\mathrm{DAB}$ as described above. Sections were then mounted onto gel-coated slides, stained with thionin, dehydrated, cleared, and coverslipped.

Data analysis. The distribution of retrogradely labeled neurons in layer IV following injections in layer III was evaluated for seven injection sites. The injection sites and labeled cells were examined under bright-field illumination. Plots of labeled neurons were obtained by tracing the injection site and charting the distribution of labeled cells using a camera lucida (magnification, $400 \times$ ). Typically cells labeled with HRP from a single injection site were distributed over several sections. Cells within $50 \mu \mathrm{m}$ of the injection site were not considered labeled by retrograde transport and were not included in subsequent analyses. Composite diagrams of two injections, illustrating the complete pattern of labeled cells, were constructed by superimposing the drawings from adjacent sections, using blood vessels, cut fibers, and laminar boundaries as landmarks. The results from three injection sites in layers IIIc and three others confined to IIIa/b were summed, and the sublaminar distribution of labeled cells in each case was quantified. Line graphs were then constructed showing the number of cells contained in each of 10 equal tangential tiers across the depth of layer IV.

Injections of HRP and biocytin in layer IV were made to examine the pattern of anterograde label in layer III. The injection sites and labeled fibers were examined using dark- and bright-field illumination. Composite diagrams of selected injections were prepared as for the retrograde transport experiments except that labeled fibers and terminals 


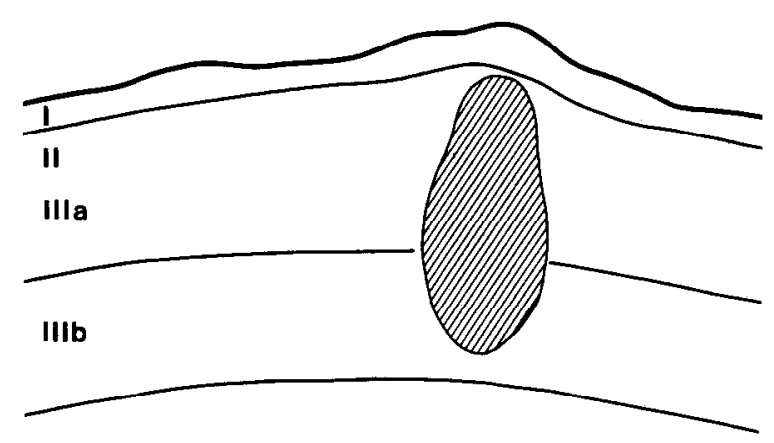

IIIC

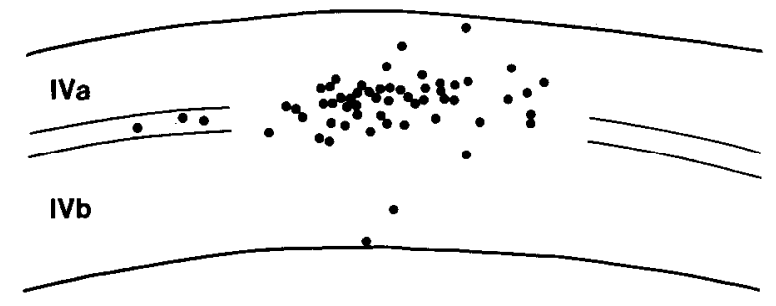

$300 \mu \mathrm{m}$
B

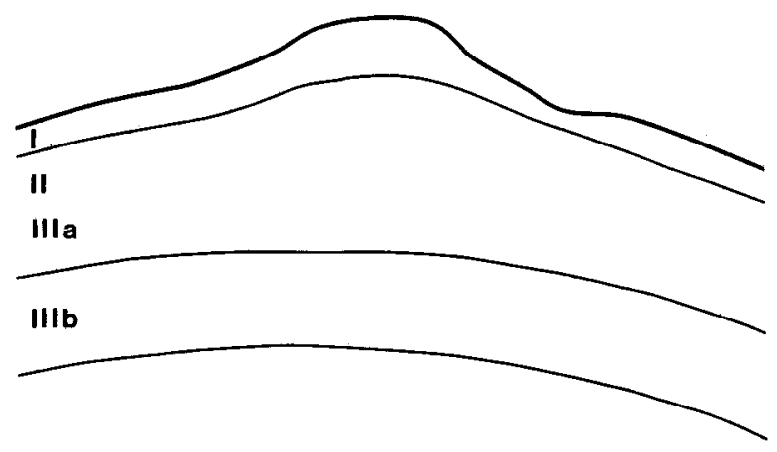

IIIC

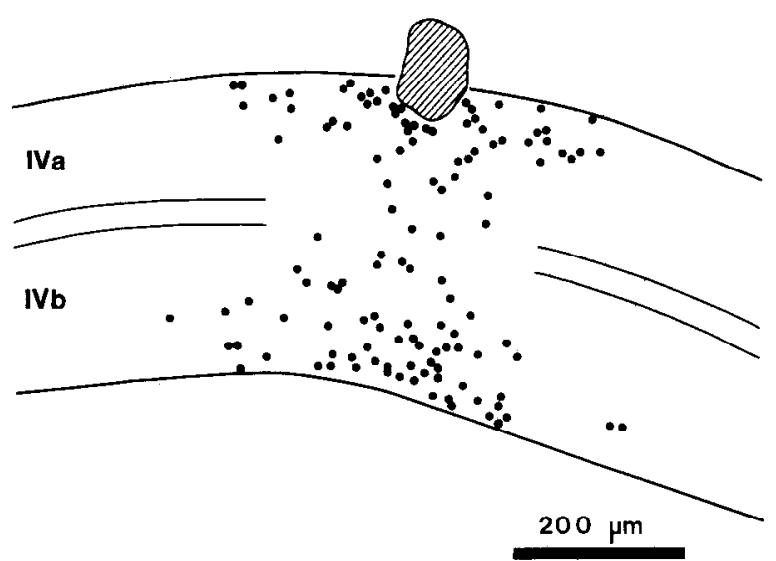

Figure 2. Two reconstructions showing the results of retrograde transport experiments. The HRP injection sites are indicated by diagonal shading and the retrogradely labeled cells are shown as black spots. A, An injection confined to layer II, IIIa, and IIIb. Labeled cell bodies are concentrated in the bottom of layer IVa and the top of IVb. B. An injection in layer IIIc. Labeled cells are concentrated at the top of IVa and bottom of IVb. Celis within $50 \mu \mathrm{m}$ of the injection sitc were not considered labeled by retrograde transport and are not illustrated here.

were drawn instead of cells. These composites document the location of label contained in every section in and around the injection site.

Small biocytin injection sites, consisting of from 1 to 20 cells, were used to examine the dendrites and axons of single cells. In most cases, there was little or no extracellular reaction product and the dendrites and axons of the cells were labeled in a Golgi-like manner. Single cells in layer IV and their axonal arbors were drawn with a camera lucida under oil immersion objectives (either $60 \times$ or $100 \times$ ).

\section{Results}

Cytoarchitecture of the tree shrew's striate cortex

Before describing the connections from layer IV to layer III, it is worth reviewing the laminar organization of the tree shrew striate cortex in Nissl-stained material (see Fig. 1). Layer IV is a prominent cell-rich layer composed of small granule cells separated into two roughly equal sublayers, IVa and IVh, by a cellsparse cleft. Layer III is composed of small- and medium-sized pyramidal cells and medium and large multipolar cells. This layer is quite wide, occupying almost half the cortical depth. It can be divided into three parts on the basis of cell density: layer IIIb, which is the most cell dense; layer IIIc, which is the least cell dense; and layer IIIa, which is intermediate. Since layers IIIa and IIIb have similar connections with layer IV, we will often refer to them collectively as layers IIIa/b. Layer II is a thin, cell-dense layer lying just beneath layer I. Its border with layer IIIa is difficult to discern, and since layer IV axons have no special relation with layer II, we have not defined this border in our figures.

\section{Retrograde transport results}

As a first step toward understanding the pattern of projections from layer IV to layer III, we made small injections of HRP at various depths within layer III and examined the pattern of cells labeled by retrograde transport within layer IV. One injection was placed in layers I and II with a small involvement of IIIa, and labeled only one cell in layer IV. Six other injections were made in layer III. Three of these involved layers IIIa and IIIb with no involvement of layer IIIc, and three were located in layer IIIc with no involvement of more superficial layers.

Regardless of the location of the injection site within layer III, labeled cells were always found in both subdivisions of layer IV. As we will show, however, the location of the labeled cells within layers IVa and IVb varied systematically with the depth of the injection site in layer III. Figure $2 A$ shows an example of an injection of HRP that involves layers IIIa and IIIb. Labeled cell bodies are located in a cluster directly below the injection site at the center of layer IV. This cluster includes cells on either side of, and within, the cleft.

Figure $2 B$ shows an injection deep in layer IIIc, involving the upper part of layer IVa. While this injection is complicated by the involvement of IVa, we chose to illustrate it because it produced the greatest number of labeled cells in layer IV. Most 


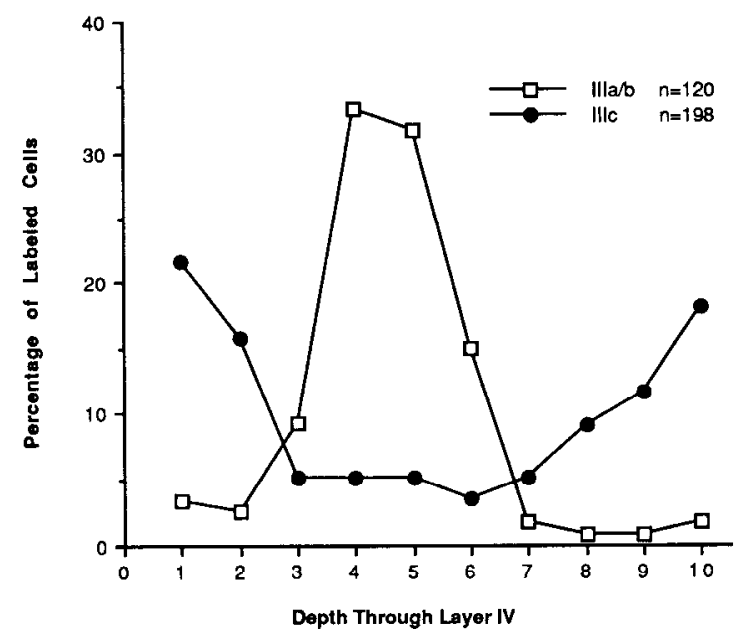

Figure 3. A line graph summarizing the distribution of labeled cells in layer IV following injections of WGA-HRP into layers IIIa/b (open squares) or IIIc (solid circles). Layer IV was divided into 10 equal horizontal tiers, and the points represent the percentage of the total labeled cells found in each tier. Injections in layers IIIa/b retrogradely label cells in the center of layer IV, while injections in layer IIIc label cells predominantly at the edges of layer IV.

of the labeled cells are located at the top of IVa and the bottom of IVb. Few labeled cells are found in the center of layer IV, and these are restricted to a small column directly under the injection site. The pattern of label in this experiment cannot be attributed to the involvement of layer IVa but rather to the involvement of layer IIIc. Injections limited to layer IVa labeled few or no cells in layer IVb, and cells labeled in IVa were restricted to a $50 \mu \mathrm{m}$ region around the injection site. The anterograde results (see below) provide further support for a projection from the edges of layer IV to IIIc.

In order to quantify the density of labeled cells at different depths in layer IV following injections of HRP into layer III, we divided layer IV into 10 equal horizontal divisions and counted the number of labeled cells in each division. We then converted these numbers to percentages and plotted them across the depth of layer IV. The combined results of three injections in layers IIIa/b and three others in layer IIIc are shown in Figure 3. The graph shows that the distribution of labeled cells from injections into layers IIIa/b peaks near the middle of layer IV (bins 4 and 5) and approaches zero at the edges of the layer. In contrast, the distribution of labeled cells following injections into layer IIIc peaks at the edges of layer IV (bins 1 and 10) and is at its lowest in the middle of layer IV.

These results provide evidence for a sublaminar organization of efferent projections from laycr IV to layer III. The upper part of IVa and the lower part of IVb appear to project primarily to layer IIIc, while the lower part of IVa and the upper part of IVb project primarily into layers $\mathrm{III} a / \mathrm{b}$.

\section{Anterograde transport results}

Given the results of our retrograde transport experiments, our next step was to make small injections of either HRP or biocytin at different depths in layer IV with the goal of tracing the projections of small groups of neurons to layer III. In general, the results obtained with both tracers were similar. However, as a rule, injections of biocytin did not label the extracellular matrix around layer IV neurons and they were often small enough to allow us to examine the dendritic morphology of the projection neurons as well as their axonal arbors. Figure 4 shows examples of small biocytin injection sites and the resultant dendritic and axonal labeling.

Projections from layer IV to layer III arise from spiny stellate neurons whose horizontally oriented dendritic fields sample from a narrow vertical extent, roughly one-third of a tier. Although smooth dendritic neurons are labeled by our injections, all of them were found to have axons that were limited to layer IV. The axons of projection cells generally exit near the bottom of the soma and, after coursing ventrally toward the white matter for $50-100 \mu \mathrm{m}$, emit from one to five recurrent branches that ascend to layer III. Collateral branches with terminal swellings in layer IV are extremely rare. In layer III, many en passant terminals are seen, and terminals on short side branches are also common. In addition, the cells in layer IV often have axon branches that terminate in layers V and VI. The arbors in the infragranular layers range from a single short axon branch to a well-developed terminal field with many branches, but in all cascs this arbor was much more sparse than the arbor in layer III. Cells at all depths of layer IV were found to contribute axonal branches to layer $\mathrm{V}$, the bulk of which were found in the upper half of $\mathrm{V}$. In order to achieve a better view of the arborizations in layer III, we have omitted the projection to the deep layers in most of our line drawings.

Projections of neurons in the lower part of I Va and the upper part of $I \mathrm{Vb}$. Figure 5 shows an example of an injection of biocytin restricted to the top part of layer $I \mathrm{Vb}$ and a small portion of the cleft. Consistent with the retrograde transport results, labeled axons extend vertically from the injection site, pass through layer IIIc, and break up into a discrete terminal field above layer IIIc. The densest zone of terminations is within layer IIIb, although a sparser network of projections extends throughout layers I-IIIa.

An injection of HRP restricted to the lower portion of IVa produced very similar patterns of label in the superficial layers, as shown in Figure 6. Injections involving the lower half of layer IVa always resulted in a dense column of vertically oriented fibers that broke up into a terminal plexus in IIIb, and a sparser collection of fibers that extended above layer IIIb all the way to the pia mater. Some labeled fibers travel horizontally within layer IIIc. These are likely due to uptake by fibers of passage (possibly from the lower part of IVb; see below), because smaller injections do not produce terminal label in IIIc.

Examples of single neurons labeled by small injections of biocytin in the lower part of IVa and upper part of IVb are shown in Figure 7. The soma of the neuron shown in Figure $7 \mathrm{~A}$ is located in layer IVa, and its dendrites extend slightly into the upper part of IVb. Many of the neurons that lie near the cleft have dendrites that sample exclusively from one or the other subdivision of layer IV (e.g., Fig. 7B) although some clearly cross the IVa-IVb border. No difference in the distribution of axon arbors was found that would correlate with whether a cell's dendrites crossed or failed to cross the cleft. The axon emerges from the ventral side of the soma, and before descending to layers V and VI where it gives rise to a terminal field (not shown), the axon gives rise to several ascending branches. These branches rise through layer IIIc with little branching until they reach the IIIb-IIIc border, where they arborize into a distinct terminal plexus. Consistent with the pattern of the bulk injection experiments, branches extend into IIIa and some continue all the way to layer I. 


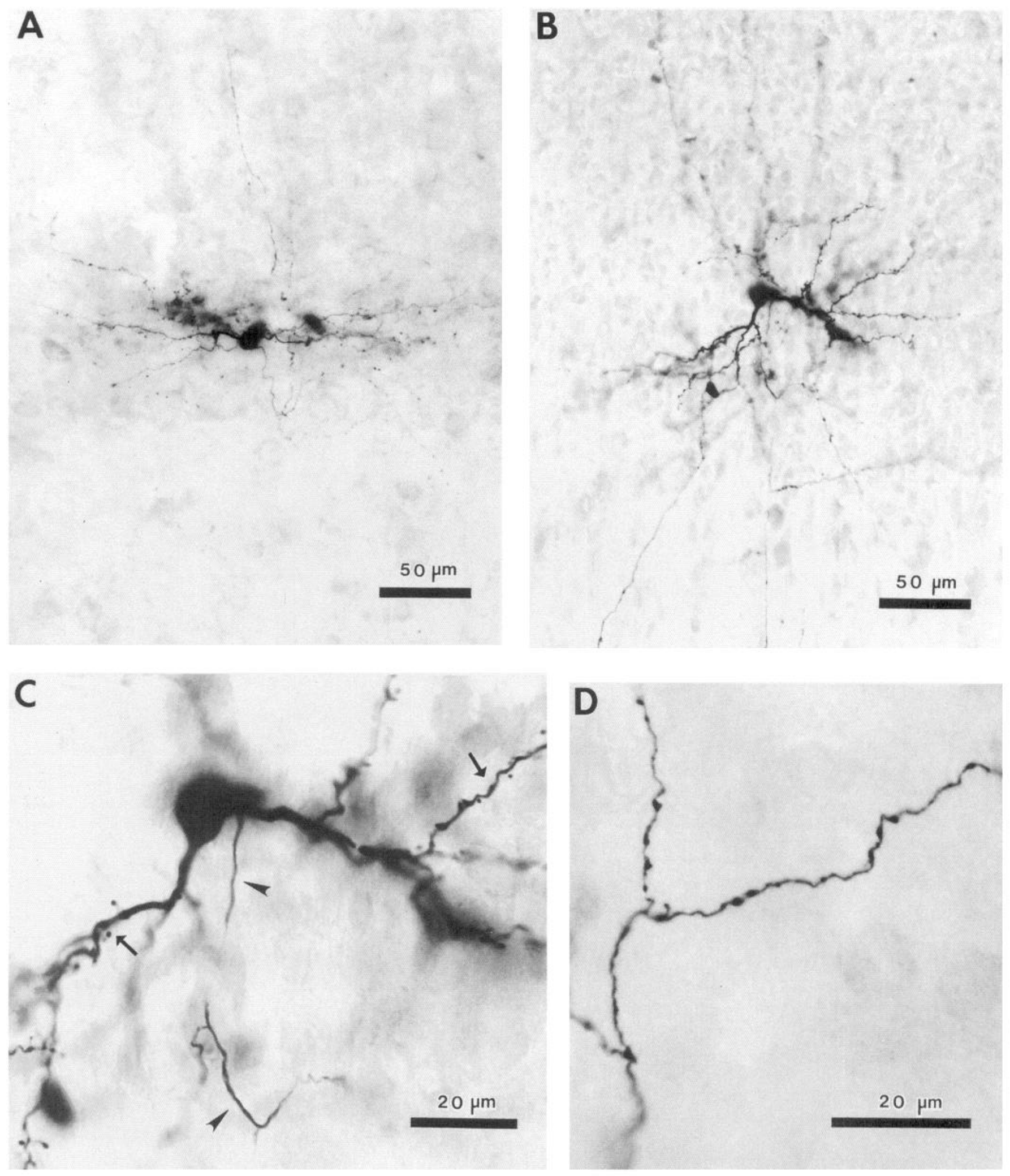

Figure 4. Examples of small extracellular biocytin injection sites and the resultant dendritic and axonal labeling. $A$ and $B$, Photomicrographs of two small injection sites in layer IV. In each there is some light extracellular reaction product and a single well-labeled cell. $C$, Higher-power photomicrograph of the cell shown in B. The axon (arrowheads) can be differentiated from the dendrites (arrows). Note the presence of dendritic spines. $D$, View of a labeled axon in layer III arising from the cell in $B$. Boutons en passant are present as well as boutons on short side branches. 

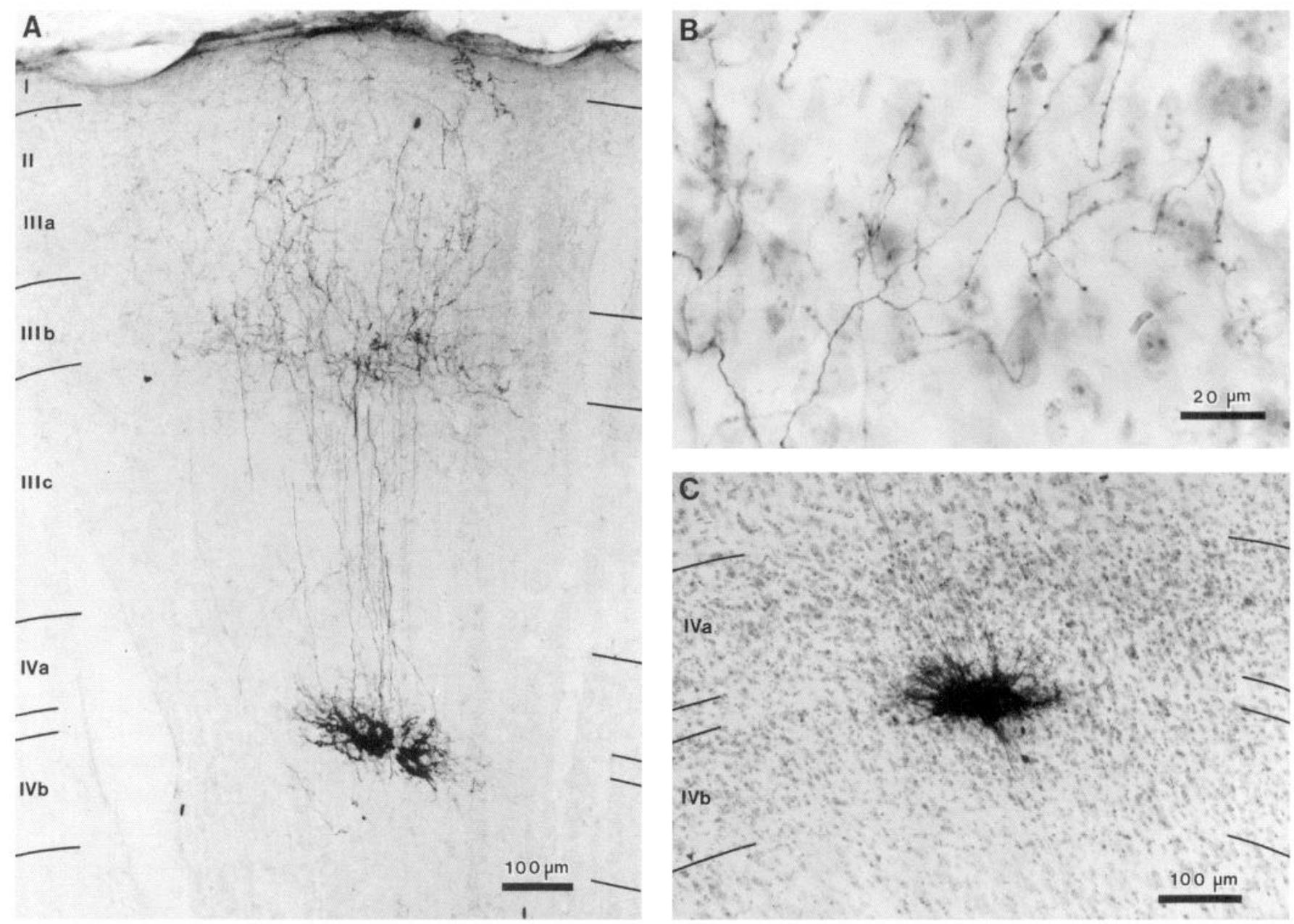

Figure 5. The axonal projections of neurons in the upper part of layer IVb and the cleft. A, A photomicrograph of an injection of biocytin located in upper layer IVb with slight involvement of the cleft. The laminar borders are indicated on the sides of the figure and are derived from the same section, which was counterstained after examination. Labeled fibers pass radially from the injection site through layers IVa and IIIc to layer IIIb, where they form a dense terminal plexus. The terminal arbor continues up into layers IIIa, II, and I, but the highest density of fibers and terminals is found in layer IIIb. B, Labeled fibers and terminals in layer IIIb shown at higher magnification. Boutons en passant and on short side spines can be clearly seen. $C$, A higher-magnification view of the injection site after counterstaining. Labeled neurons are located in the upper part of layer $\mathrm{IVb}$, with the exception of one labeled cell in the cleft. There is no involvement of layer IVa.

Projections of neurons in the upper part of layer IVa and the lower part of $I \mathrm{Vb}$. Injections of biocytin or HRP that are restricted to the upper part of IVa or the lower part of IVb result in labeled terminals that are largely restricted to layer IIIc. An example of a biocytin injection into the upper part of layer IVa is shown in Figure 8. Note the scarcity of vertically directed fibers rising through IIIc into IIIb, like those found after injections in lower IVa and upper IVb. Most of the fibers are concentrated immediately above the injection site in the lower part of layer IIIc.

Examples of two single neurons reconstructed from smaller extracellular injections of biocytin in the upper part of layer IVa are shown in Figure 9. These spiny stellate cells have horizontally oriented dendritic arbors that are restricted to the upper one-third of IVa. The main axonal trunk exits from the ventral surface of the cell and descends to layer $\mathrm{V}$ where it gives rise to a sparse terminal arbor (not shown). Several collaterals arise from this descending branch and ascend to layer III where they branch repeatedly in the lower part of layer IIIc.
An example of an HRP injection into the lower part of layer IVb is shown in Figure 10. Labeled fibers extend vertically from the injection site and pass through the more superficial parts of layer IV before they break up at the border between layer IVa and layer IIIc. The lower part of IIIc is densely filled with terminals, and only occasional fibers extend into the upper portion of IIIc.

Figure 11 shows two examples of neurons in the lower part of IVb that were filled with small extracellular injections of biocytin. The dendrites of these cells sample from roughly the bottom third of layer IVb and the single descending axon gives rise to collateral branches that ascend to the IIIc-IVa border and arborize in a terminal field that covers the lower half of layer IIIc.

Projections of neurons in the middle of layer IVa and the middle of $I V b$. The HRP and biocytin evidence presented so far has suggested that layer IVa and IVb each can be divided into two zones, upper and lower, that differ in their pattern of projection to layer III. This conclusion is based on a comparison 


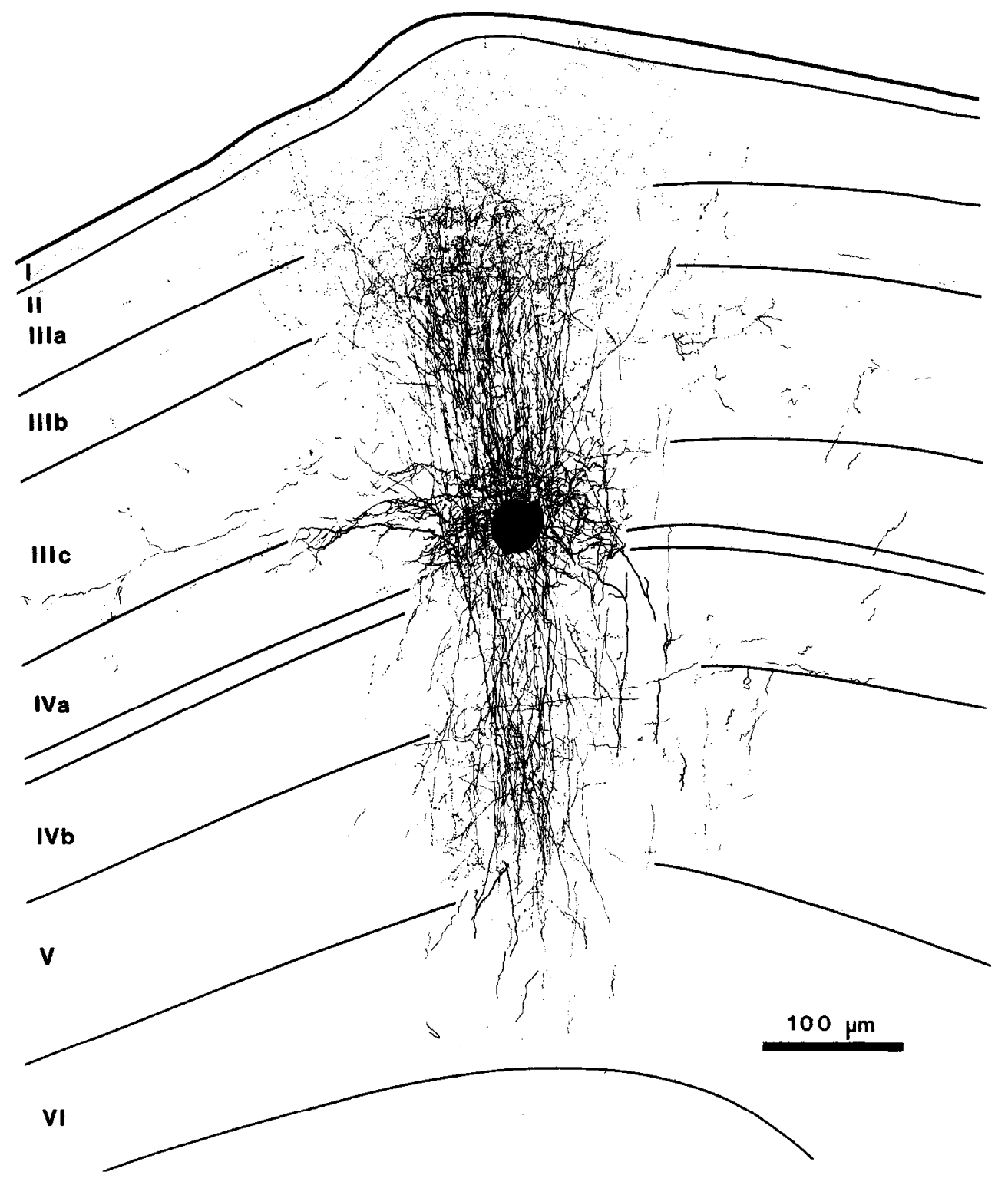

Figure 6. A reconstruction of the pattern of label resulting from an HRP injection into the bottom of layer IVa. The injection site is shown as the large black spot; labeled fibers and terminals are shown as lines and dots, respectively. Labeled fibers rise through IIIc to terminate heavily in layer IIIb. A lighter terminal field is present in IIIa with sparse terminals in layers II and I. There are also labeled fibers descending from the injection sitc. Somc of thesc appear by their caliber and morphology to be thalamic afferents labeled by retrograde transport, while others are cortical in origin and terminate in the infragranular layers. of the projections of neurons that lie near the upper and lower borders of each layer. Left open is the issue of the projections of neurons that lie in the center of IVa and IVb. Our ability to confine biocytin to a very small number of neurons made it possible to label cells in the middle of layers IVa and IVb and to compare the projections of these cells with those above and below them. Figure $12 A$ shows a line drawing of an injection of biocytin that labeled four cells in the middle of layer IVb. The dendritic processes of these cells occupy the middle third of layer IVb, and their axonal arbors exit from the ventral surface and extend branches upward through IVa and the bottom half of layer IIIc before terminating in the upper part of IIIc. The projections of these neurons were limited to the upper part of IIIc and did not encroach on either the lower part of IIIc or IIIb (compare this pattern with the pattern of label shown in Figs. 5 and 8).

Figure $12 B$ shows an injection of biocytin that labeled two neurons in the middle of IVa. Like their counterparts in the middle of IVb, the axons of these neurons have terminal fields that arborize in the upper part of layer IIIc, leaving the lower part of IIIc and IIIa/b virtually devoid of axon terminals.

\section{Discussion}

Our results show that the spine-bearing stellate neurons in layer IV project throughout the depth of the overlying cortical layers (layers I-IIIc). The projections of the two subdivisions of layer IV (IVa and IVb) overlap throughout this region and do so in a highly stratified, mirror-symmetric fashion that respects the vertical position of neurons within each sublayer: neurons located in upper IVa and lower IVb have overlapping terminal fields in the lower part of layer IIIc, neurons in the middle of IVa and IVb have overlapping terminal fields in upper IIIc, while neurons in the lower part of IVa and upper IVb have overlapping terminal fields in layers IIIa/b (Fig. 13B). In the following sections, we consider these findings in the light of other studies of tree shrew striate cortex, and we speculate on their significance for understanding the functional organization of visual cortex. 

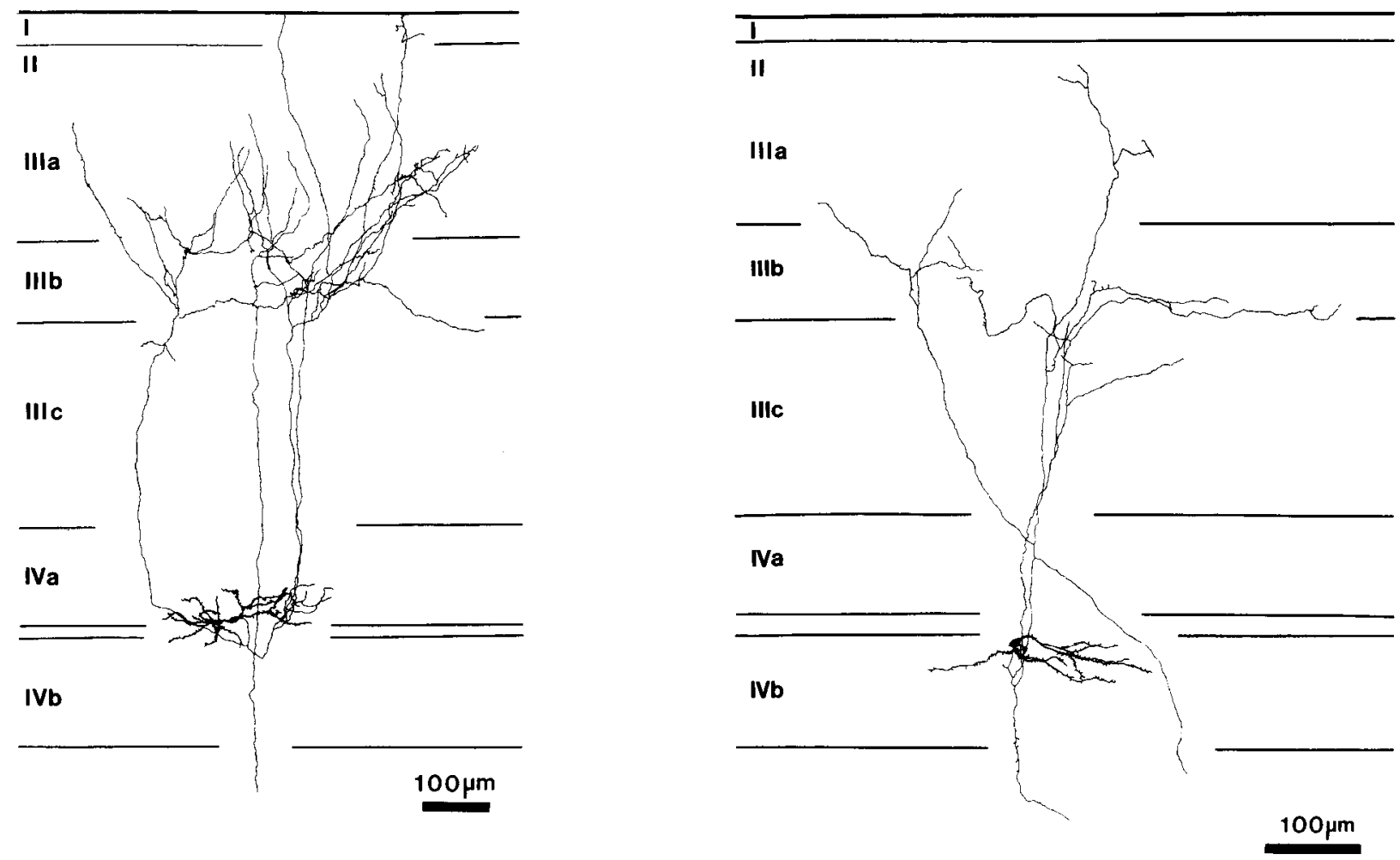

Figure 7. Two spiny stellate cells labeled and reconstructed after small injections of biocytin in the bottom of IVa $(A)$ or top of IVb $(B)$. The cells' axons emerge from the bottom of the soma and descend for about $50 \mu \mathrm{m}$ before giving rise to collaterals. These ascend through layer IV and IIIc to layer IIIb, where they arborize and form terminal fields that are densest in IIIb but continue up through layers IIIa, II, and I. In addition, the axons descend to layers V and VI, where they give rise to a modest terminal field. These fields have been omitted in the drawings to preserve the detail of the layer III arbor.

Comparisons with previous descriptions of layer IV neurons in tree shrew striate cortex

The only other description of the axonal projections of layer IV neurons in the tree shrew striate cortex is that contained in the review chapter of Lund et al. (1985). This report contains examples of several Golgi-filled spiny stellate neurons that support some of the results we have described. They show two spiny stellate neurons in the upper half of IVa whose axons arborize in layer IIIc but do not extend to layer IIIb. Also consistent with our findings is their illustration of a spiny stellate cell, which was located in the upper half of IVb, whose axon extends into layer IIIb without arborizing in IIIc.

Lund et al. (1985) also observed a type of spinous cell projecting to layer III that we have not described: a pyramidal cell whose soma is located in the cleft or in the upper part of layer $\mathrm{IVb}$ and whose apical dendrite ascends through layer IV and IIIc to arborize in layer IIIb. We have succeeded in labeling only a few of these pyramidal neurons in our biocytin experiments, and while we can confirm their location and the distribution of their apical dendrites, their axonal arbors were not filled sufficiently to determine their termination pattern. The report by Lund et al. (1985) indicates that the axons of these cells arborize in layers IIIa/b, in the same region as the apical dendrites, and this would certainly be consistent with the results of our bulk labeling experiments. Thus, the axonal projections of these pyramidal cells are likely to resemble those of the spiny stellate cells that lie at the same depth in layer IV.

All of the neurons that we identified as having axons that project to the superficial layers were spinous, a result that may seem somewhat surprising since smooth dendritic neurons in layer IV of other species have been shown to project to many of the targets of the spiny stellate population (e.g., Lund, 1987). The lack of projections from smooth dendritic neurons cannot be explained by a failure of biocytin to label this population: our biocytin injections filled a variety of smooth dendritic layer IV neurons, but all of these had axonal arbors that were confined to this layer. Lund et al. (1985) also observed several different types of smooth and dendritic neurons with axon arbors confined to layer IV, but in addition, they found some smooth dendritic bipolar neurons whose dendritic and axonal processes extended vertically out of layer IV, reaching as high as layer III and as deep as layer VI. We have not yet encountered this type of neuron in our biocytin material, which suggests that it makes a rather small contribution to the total population of layer IV terminals in the superficial layers. Nevertheless, this raises the 

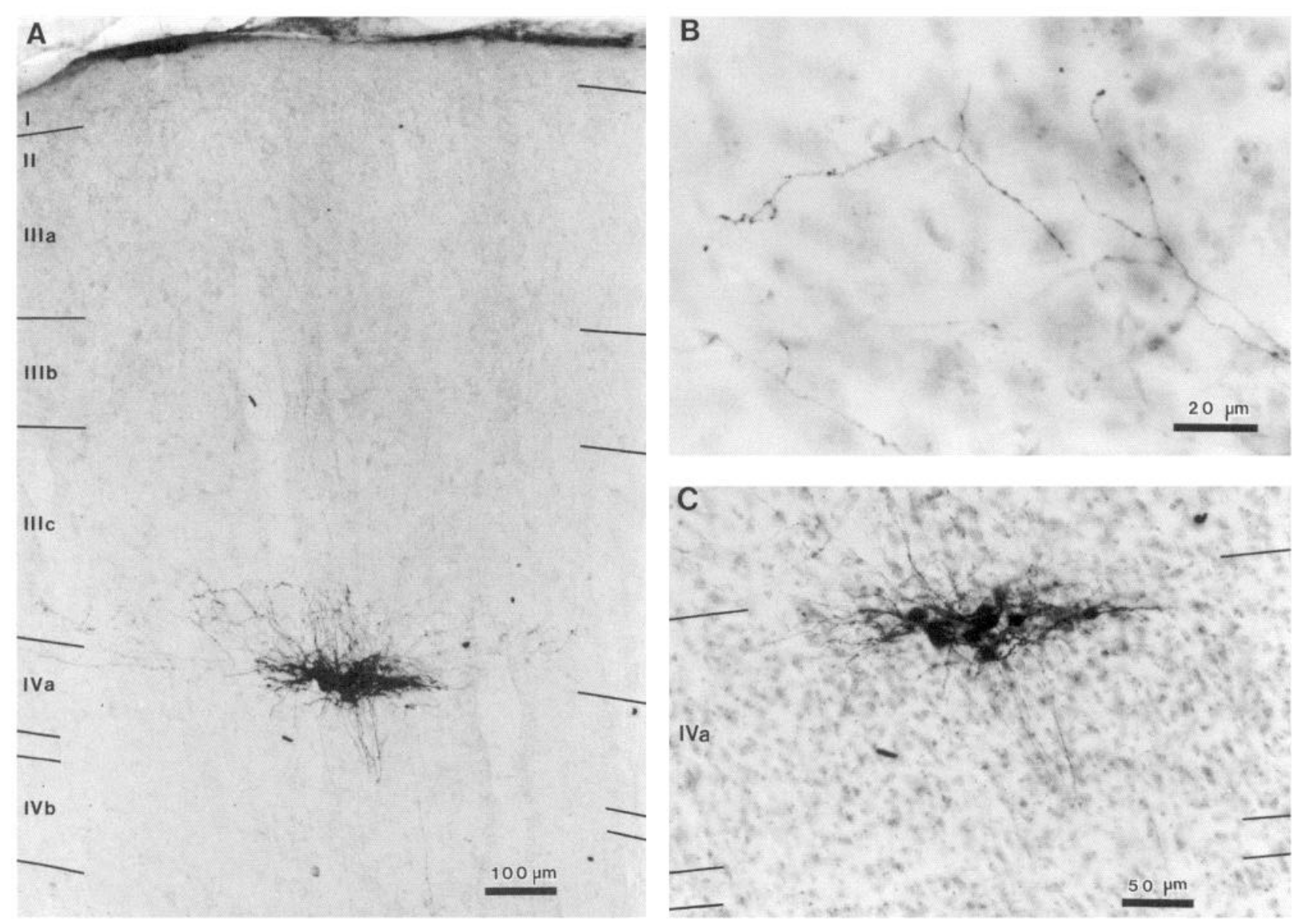

Figure 8. The axonal projections of neurons in the upper part of layer IVa. A, Photomicrograph of an injection of biocytin into the upper part of layer IVa. The laminar borders are indicated on the sides of the figure and are derived from the same section, which was counterstained after examination. Labeled fibers descend toward the cleft, where they turn and proceed into layer IIIc. Once in IIIc, they arborize into a terminal field in the bottom half of the layer. Labeled fibers do not pass into more superficial layers. $B$, Labeled fibers and terminals in lower layer IIIc shown at higher magnification. $C$, A higher-magnification view of the injection site after counterstaining the section. Labeled cells are restricted to the top third of layer IVa.

possibility that small populations of neurons with patterns of projections that differ from those we illustrated could have escaped detection.

\section{Projections from layer IV to layer III and their relation to $O N / O F F$ and binocular convergence}

Our reason for studying the projections of neurons in layers IVa and $\mathrm{IVb}$ was to determine what happens to the ON and OFF pathways beyond the first cortical synapse. On the basis of the patterns of LGN projections to layer IV, we had suggested that ON/OFF and binocular convergence in the tree shrew might be accomplished in a two-stage process. In the first stage, the ON pathways from the two eyes are combined in layer IVa and the OFF pathways from the two eyes are combined in layer IVb; in the second stage, binocular ON and OFF pathways are brought together via the projections from layer IV to the superficial layers. The demonstration that the neurons in IVa and IVb have overlapping axonal distributions throughout the superficial layers makes it likely that these projections bring about the convergence of the ON and OFF pathways onto individual neurons in layer III. Physiological studies of neurons in the superficial layers also provide support for the view that the ON and OFF pathways that are segregated in layer IV (Kretz et al., 1986; Raczkowski and Fitzpatrick, 1990) converge onto individual neurons in the superficial layers. For example, Humphrey et al. (1977) noted that most neurons in layer III have simple and complex receptive field properties similar to those described in other species, and our own unpublished results show that most neurons in the superficial layers exhibit both $\mathrm{ON}$ and OFF responses.

What we could not have anticipated is the remarkable laminar specificity in the projections from layer IV to layer III that brings together the axons from neurons that lie at mirror-symmetric locations in layers IVa and IVb. It seems likely that the significance of this pattern lies in response properties other than $\mathrm{ON}$ and OFF that vary in a mirror-symmetric fashion across the depth of layers IVa and IVb. One likely candidate for variation with depth is ocular dominance. As shown in Figure 13A, LGN neurons that respond to stimulation of the contralateral eye give rise to fibers that terminate across the entire depth of IVa or $\mathrm{IVb}$ (depending on receptive field sign), while those that respond to stimulation of the ipsilateral eye have projections that are restricted to the upper half of IVa or the lower half of IVb 
A

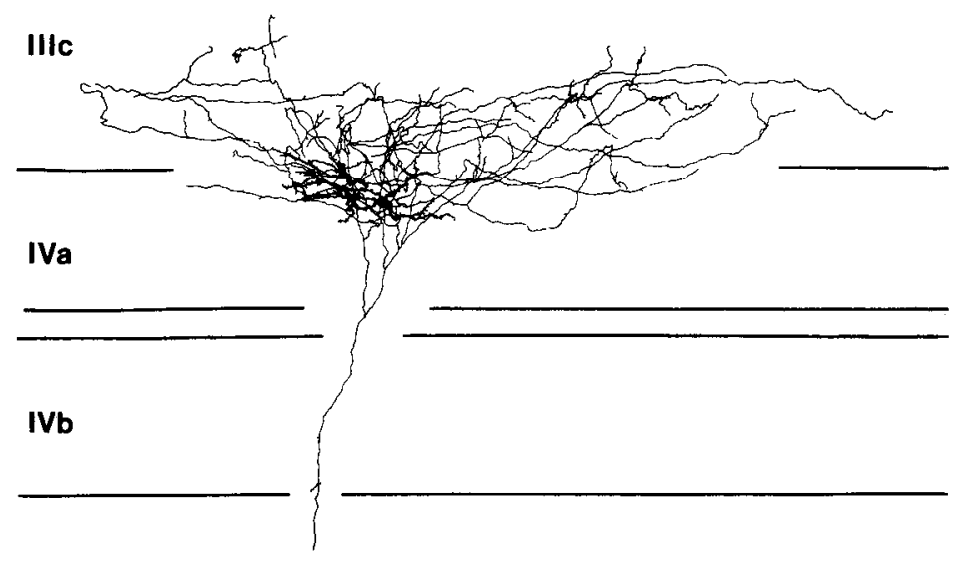

B

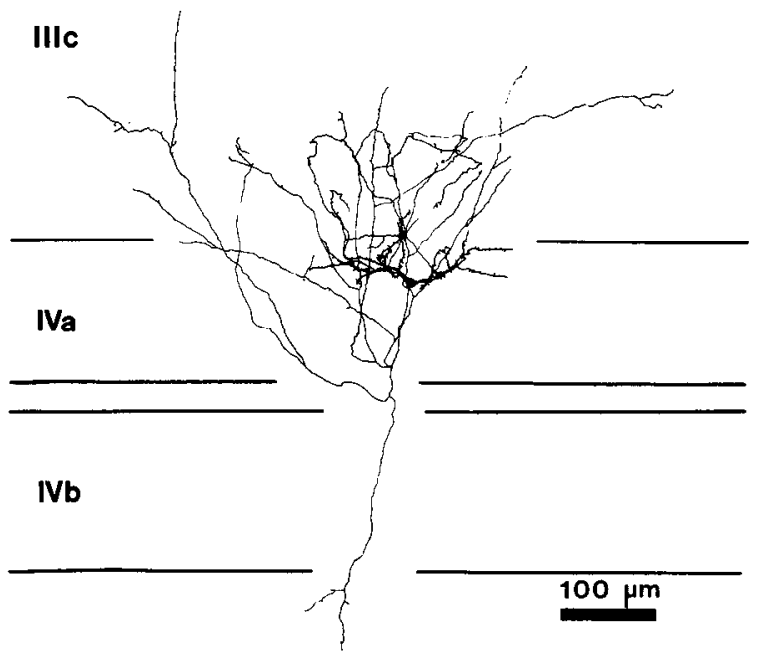

Figure 9. Two spiny stellate cells $(A$ and $B)$ in the top of layer IVa labeled by small injections of biocytin. The axons descend initially before sending several collaterals back to layer IIIc, where they arborize in the bottom half of the layer. One axon branch from each cell continues to layer $\mathrm{V}$, where it gives rise to a sparse arbor that is not fully illustrated here.

(Casagrande and Harting, 1975; Hubel, 1975; Conley et al., 1984; Raczkowski and Fitzpatrick, 1990). Golgi impregnation studies (Geisert and Guillery, 1979; Lund et al., 1985) and our own work using injections of biocytin reveal that layer IV spiny neurons have dendritic fields that are horizontally oriented, sampling from a very narrow vertical dimension of the layer. Based on the anatomy, one would expect to find a gradation in ocular dominance with strongly binocular neurons near the edges of layer IV, and neurons driven almost exclusively by the contralateral eye near the center. Consistent with this view, multiunit recordings have shown binocular responses throughout IVa and IVb, except for a region in the middle of layer IV, which contained neurons that responded only to the contralateral eye (Kretz et al., 1986).

It should be noted that extracellular single unit recordings of Humphrey et al. (1977), while demonstrating that many of the neurons in layer IV are binocular, found no clear-cut relationship between the ocular dominance of a neuron and its depth within layer IV. How the results of these two physiological studies can be reconciled is difficult to say. Perhaps the negative results of Humphrey et al. (1977) could be explained by ambiguities inherent in assessing the location of single units with extracellular recordings. For example, there is significant mixing of the axons of neurons from different depths within layer IV (almost all of the neurons that we encountered send their axons toward the white matter and then send off collaterals that turn and rise into layer III) and this could obscure a systematic representation of ocular dominance. While the physiological evidence is equivocal, there is no evidence for structural features such as dendritic sampling or axonal projections within layer IV that would degrade the partial segregation of geniculate axons driven by the ipsilateral and contralateral eye.

With these considerations in mind, we suggest that the highly stratified projections from layer IV to layer III serve to unite $\mathrm{ON}$ and OFF inputs that have matching ocular dominance char- acteristics. We recognize that ocular dominance may not be the only property that varies across the depth of IVa and IVb. While there is no evidence for other functional differences within the populations of LGN neurons supplying layer IV that would warrrant their parcellation into classes such as " $\mathrm{X}$ " or " $\mathrm{Y}$ " (Conway and Schiller, 1983; Raczkowski and Fitzpatrick, 1990; T. T. Norton, personal communication), it is possible that response properties generated within layer IV such as orientation selectivity, endstopping, and so on, may also vary in a graded fashion across these sublayers. Even so, our findings lead to some specific predictions about the distribution of ocular preferences across the depth of layer III. We would expect to find strongly binocular neurons in the lower part of IIIc, neurons driven almost exclusively by the contralateral eye in layers II and IIIa/b, and neurons with ocular dominance characteristics intermediate between these extremes in the upper part of IIIc. Although the study of ocular dominance in the tree shrew by Humphrey et al. (1977) made no attempt to look for systematic differences across the depth of layer III, they did note that the cells in layer III most strongly influenced by the ipsilateral eye were located within $120 \mu \mathrm{m}$ of the upper border of layer IV. This corresponds to the lower part of IIIc, the region that receives overlapping projections from neurons in the bottom of IVb and the top of IVa.

It is worth noting that layer IV is not the only source of visual projections to layer III. In the tree shrew, as in other species, small neurons in the W-like layers of the LGN and pyramidal neurons within cortical layer $\mathrm{V}$ also send projections to layer III (Lund, 1973; LeVay and Gilbert, 1976; Fitzpatrick et al., 1983; Conley et al., 1984; Martin and Whitteridge, 1984; Usrey et al., 1992). Based on relative densities, the projections from layer IV are likely to provide the main excitatory drive to neurons in layer III; however, the contribution of these other sources to the $\mathrm{ON} / \mathrm{OFF}$ and binocular responses of layer III neurons remains unknown. 


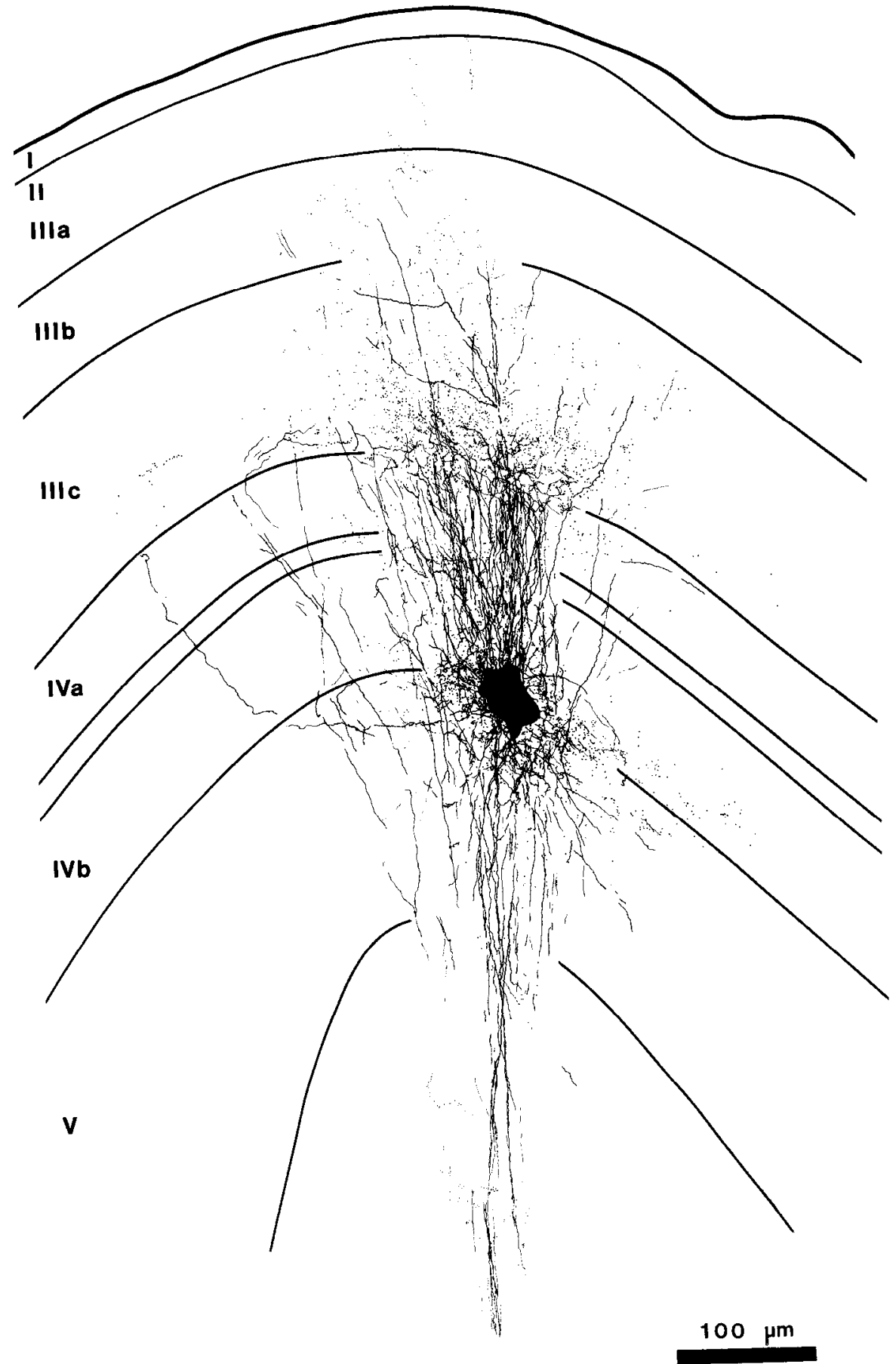

Figure 10. Reconstruction of the label after an injection of HRP that involves the lower part of layer IVb. The injection site is shown as the large black spot; labeled fibers and terminals are shown as lines and dots, respectively. Labeled fibers rise up through layer IV to terminate in layer IIIc, predominantly in the deepest part.

Role of serial order and spatial gradients in $\mathrm{ON} / \mathrm{OFF}$ and binocular convergence

From the present results and previous work describing the organization of connections within the striate cortex of the tree shrew, we suggest that two features, (1) the serial order of convergence (eye before sign) and (2) the generation of parallel ocular dominance gradients for the ON and OFF pathways, serve specific roles in meeting the requirements of ON/OFF and binocular convergence described earlier (see introductory remarks). Combining eye before sign is an economical way to match the spatial inputs from the two eyes while at the same time generating a variety of different types of receptive fields (see also Fitzpatrick and Raczkowski, 1990). We assume that the binocular neurons present in layer IV have receptive fields in the two eyes that are spatially matched. Once this matching has been accomplished, no matter how these single sign neurons converge onto individual layer III neurons, the receptive fields that are generated will have the same spatial organization in the two eyes. Binocular ON and binocular OFF neurons in layer IV can thus be viewed as "building blocks" that could be combined in a variety of ways to generate different receptive field types (i.e., simple or complex) in layer III. The economy of bringing together eye before sign can be appreciated by comparing it with 

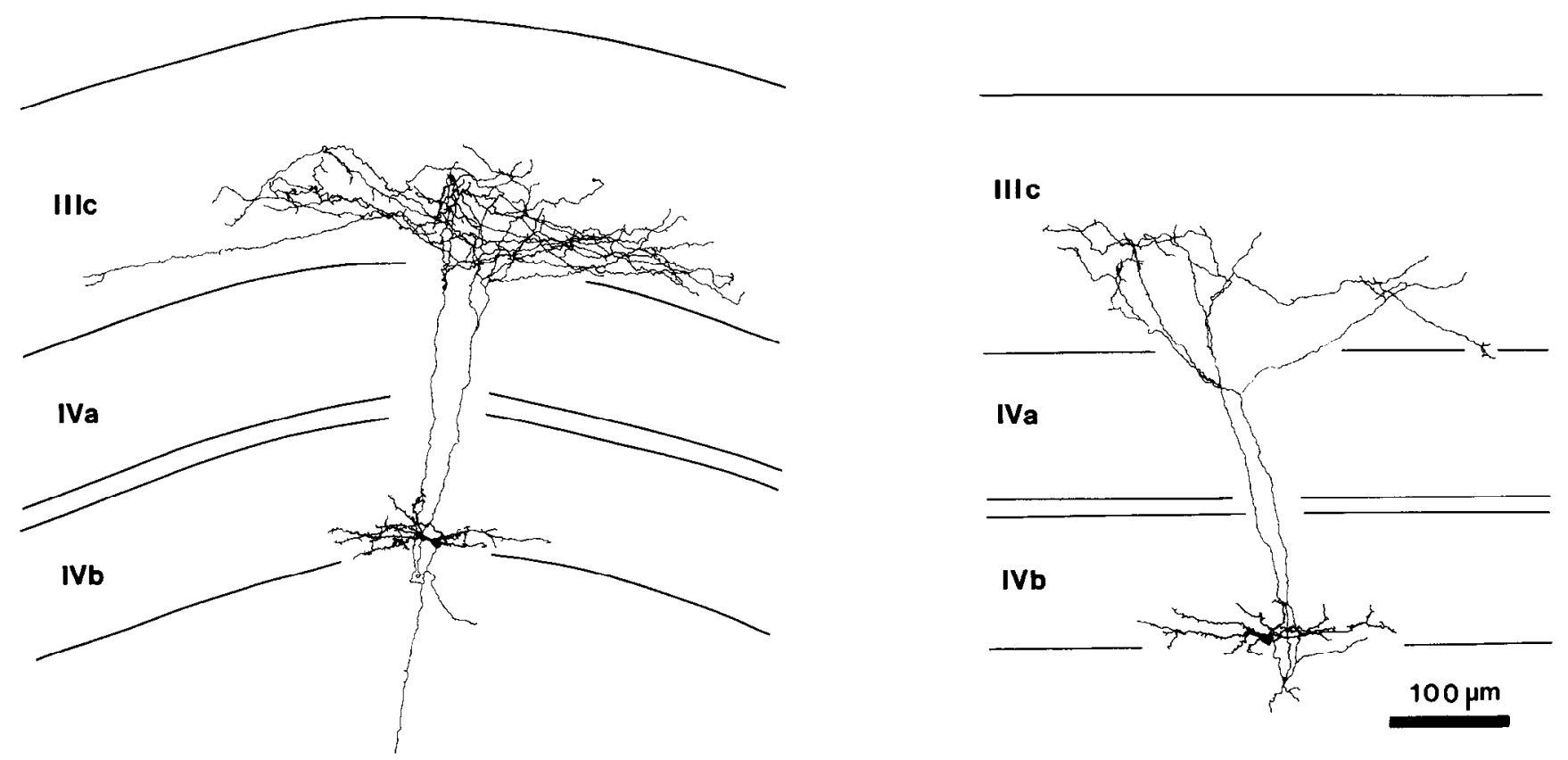

Figure 11. Two spiny stellate cells $(A$ and $B)$ in the bottom of layer IVb labeled by small injections of biocytin. The axons descend initially before sending several collaterals back to layer IIIc, where they arborize in the bottom half of the layer.

the alternative sequence, combining sign before eye. The latter approach requires that each spatial arrangement be generated twice, once for each eye, and then that monocular neurons with matching spatial characteristics converge quite precisely to create binocular ones.

The order of convergence, by itself, however, provides no constraints on the ocular dominance of layer IV neurons that converge onto individual neurons in layer III. For example, there is nothing about the order of convergence that would prevent the mixing of information from ON neurons that are driven equally well by the right and left eye with information from OFF neurons that are driven only by the contralateral eye. We would argue, however, that the presence of parallel spatial gradients for binocularity, one for the ON pathway and another for the OFF pathway, reduces the chances of such a mismatch. By bringing together the axons from neurons that lie at corresponding depths in layer IVa and IVb, the tree shrew meets the second requirement for ON/OFF and binocular convergence: generating cells with a range of ocular dominance properties while ensuring that the relative strength of input from the two eyes is the same for both signs.

Many of the aspects of cortical circuitry that we have describcd in this articlc arc unique to the tree shrew. For example, there is no evidence from work in other species that ON center and OFF center LGN axons are segregated in a laminar fashion, nor is there evidence for a laminar stratification of LGN axons driven by the left and right eyes. Since ON/OFF and binocular convergence occur in other species, laminar stratification cannot be essential for this process. And yet the two features of cortical circuitry that we have identified as important for ON/OFF and binocular convergence in the tree shrew-serial order and spatial gradients - are themselves not dependent on lamination. The question that arises, then, is whether it is possible, even without laminar stratification, for serial order and spatial gradients to play a role in ON/OFF and binocular convergence in other species.

\section{Sequential convergence in other species}

Without lamination as a clue, it is much more difficult to determine the extent to which serial order plays a role in ON/OFF binocular convergence. For example, in the cat, many binocular $\mathrm{ON} / \mathrm{OFF}$ neurons are present in layer IV and most of these can be activated monosynaptically by electrical stimulation of the LGN (Bullier and Henry, 1979; Martin and Whitteridge, 1984). At first glance, these findings suggest that instead of serial processing, both forms of convergence occur simultaneously. Even in the ferret (Zahs and Stryker, 1988) and the mink (LeVay et al., 1987) where the terminal fields of ON and OFF geniculate axons terminate in separate columns, the presence of binocular ON/OFF neurons in layer IV weighs heavily in favor of a simultaneous convergence mechanism. Yet the demonstration of monosynaptic LGN input to a binocular ON/OFF neuron does not necessarily mean that all four classes of LGN fibers contact this neuron directly. Thus, it would be difficult to rule out the possibility that a binocular ON/OFF cell in layer IV receives dircct $O N$ inputs from the LGN and complementary OFF inputs via a disynaptic pathway within layer IV. In the cat, layer IV spiny stellate cells have extensive axonal arbors within layer IV (Gilbert and Wiesel, 1979; Martin and Whitteridge, 1984; Callaway and Katz, 1992), and these arbors could be critical for the formation of the binocular ON/OFF cells. Furthermore, some of the neurons in layer IV of the cat and the mink are binocular, but they have strictly ON or OFF receptive fields (LeVay et al., 1987), so it is possible that the axons of these neurons could converge in a sequential manner like that suggested for the tree shrew. 


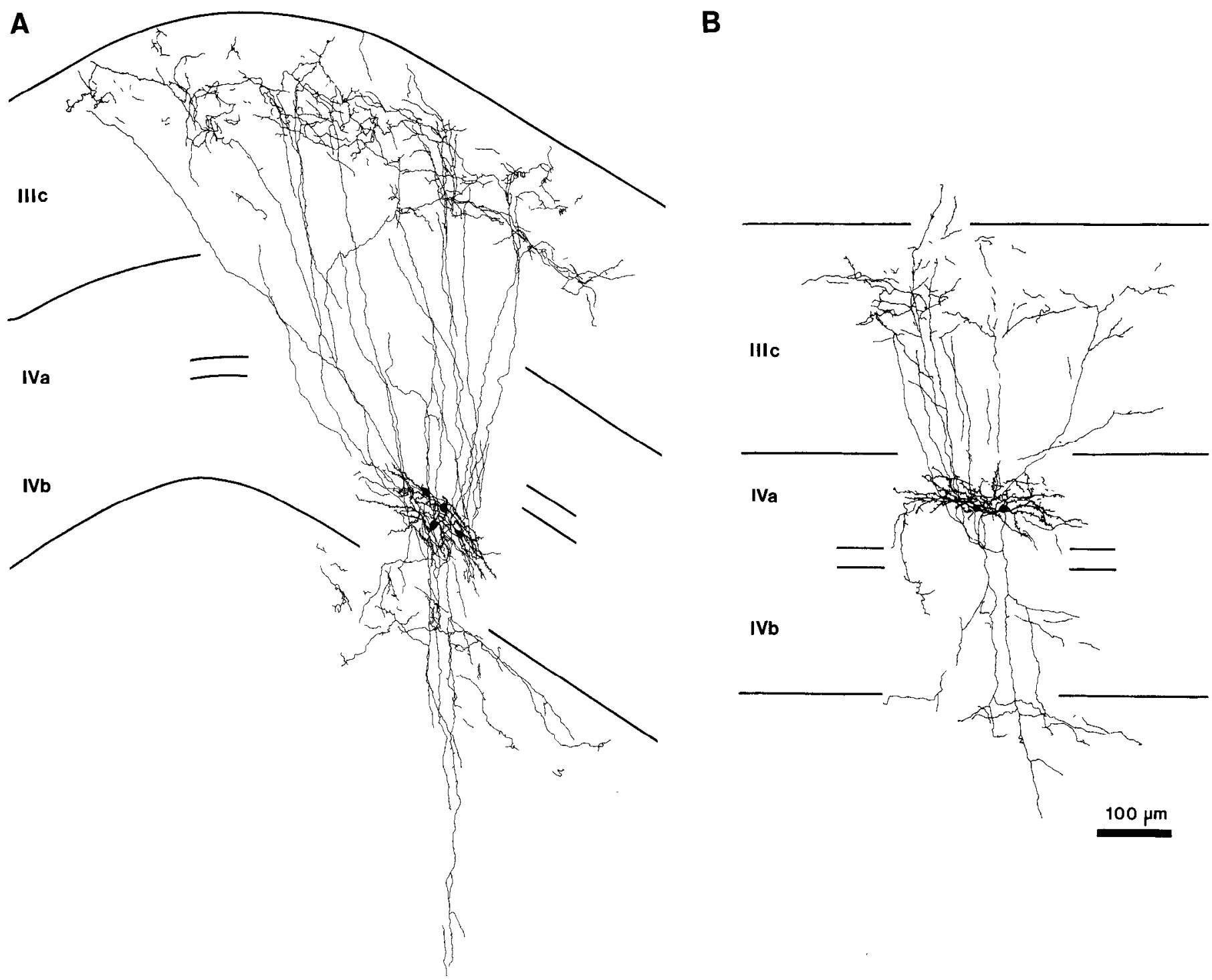

Figure 12. Projections from layer IV to the upper part of layer IIIc. $A$, Line drawing depicting the results of a small injection of biocytin restricted to the middle of layer IVb. The four spiny stellate cells labeled by this injection give rise to axon arbors that are restricted to the upper part of layer IIIc. All of the axons labeled by this injection are depicted. $B$, Line drawing depicting the results of a small injection of biocytin restricted to the middle of layer IVa. The two spiny stellate cells labeled by this injection give rise to axon arbors that are restricted to the upper part of layer IIIc. All of the axons labeled by this injection are depicted.

In the monkey, any attempt to consider the mechanisms of ON/OFF and binocular convergence must take into account the differences in the intracortical circuitry and the receptive field properties associated with the magnocellular and parvocellular layers of the LGN. The available evidence suggests that the magno stream uses a serial convergence scheme for ON/OFF and binocular convergence but, unlike the tree shrew, the sequence involves mixing the information from the $\mathrm{ON}$ and $\mathrm{OFF}$ pathways before mixing the inputs from the two eyes. A significant number of the neurons in IVC $\alpha$ (the target of magnocellular afferents) are monocular simple cells with mixed $\mathrm{ON}$ and $\mathrm{OFF}$ responses (Bullier and Henry, 1980; Livingstone and Hubel, 1984). Neurons in IVC $\alpha$ send their axons to layer IVB (Lund, 1973; Fitzpatrick et al., 1985), a layer that contains binocular neurons (Hubel and Wiesel, 1977). The simplest interpretation of these findings is that monocular ON/OFF neurons in IVC $\alpha$ converge to generate binocular ON/OFF neurons in IVB. In contrast, at the first cortical synapse in the parvo stream, neither ON/OFF nor binocular convergence seems to occur: neurons in IVC $\beta$ have receptive field properties that are identical to those in the parvocellular layers of the LGN (Blasdel and Fitzpatrick, 1984). Neurons in layer IIIb, the principal target of neurons in IVC $\beta$ (Fitzpatrick et al., 1985), exhibit both binocular receptive fields and mixed ON and OFF responses (Hubel and Wiesel, 1977). Whether ON/OFF convergence occurs with binocular convergence as a single event or as a serial operation within IIIb is unclear.

Given the variation that exists between species and even, perhaps, between streams in the macaque, it seems safe to conclude that there is no single rule for the order in which ON/ OFF and ocular inputs are combined. The three possible ways of mixing eye and sign, simultaneous convergence, eye before sign, and sign before eye all seem to be represented. It remains to be determined whether the existence of different forms of 


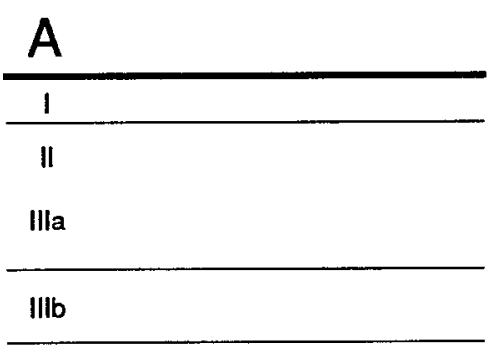

Figure 13. A, Summary of the projections from the LGN to cortical layer IV. Axons from ON center geniculate cells terminate in layer IVa, and axons from OFF center geniculate cells terminate in layer IVb. Geniculate axons driven by the contralateral eye terminate throughout layers IVa and IVb, while those driven by the ipsilateral eye terminate in the top of IVa and bottom of IVb. $B$, Summary of the projections from layer IV to supragranular layers. Cells at the edges of layer IV, that is, at the top of IVa and the bottom of IVb, project to the bottom of IIIc. Cells in the center of IV, that is, at the bottom of IVa and top of IVb, project strongly to layer IIIb and less densely to layers IIIa, II, and I. Cells in the middle of $\mathrm{IVa}$ and the middle of $\mathrm{IVb}$ project to the top of layer IIIc.

V

VI

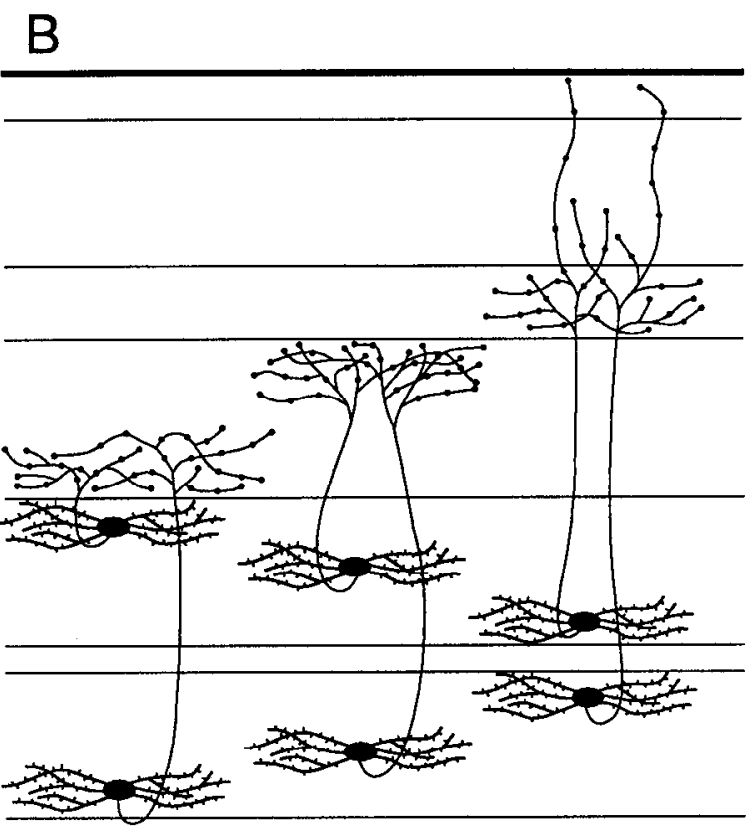

convergence simply reflects the evolution of different solutions to the same problem, or whether there are other functional requirements that dictate the form of convergence that is used in each case.

\section{Spatial gradients in other species}

The use of spatial gradients for bringing together the inputs from the two eyes seems to be a mechanism common to a wide variety of mammals. In cats and many primates, mixing of the inputs from the two eyes begins with a segregated pattern of LGN axons in layer IV followed by a merger of these pathways, either within layer IV (in the cat) or via projections of layer IV cells to more superficial layers (in the monkey), such that there is an orderly progression from dominance by the ipsilateral eye, to equal binocular input, to dominance by the contralateral eye (Hubel and Wiesel, 1972, 1977; Shatz and Stryker, 1978). The obvious difference between the tree shrew and other species that have been examined is the dimension of the cortex across which this gradient is distributed. In most species, the gradient is distributed across the surface of the cortex and is repeated at roughly $1 \mathrm{~mm}$ intcrvals. Our evidence suggests that ocular dominance gradients in the tree shrew are established across the depth of the cortex, twice in layer IV and once again across layers II and III.

We would argue that regardless of the dimension across which they are organized, spatial gradients in ocularity are an efficient means to generate ocular dominance variation while at the same time ensuring that the relative strengths of the inputs from the two eyes are the same for both signs. In monkeys, the basis for ocular dominance gradients is the segregation of LGN axons driven by the left and right eye into alternating $500-\mu \mathrm{m}$-wide slabs in layer IVC (Hubel and Wiesel, 1977). The ocular dominance preferences of neurons outside layer IV reflect this arrangement in that cells that are most binocular are located at the borders of the slabs in layer IV, whereas those dominated by one eye are located near the centers of these slabs. If we consider the connections related to the parvo stream, binocular mixing is likely to be accomplished by the projection of layer IV cells beyond the edges of the ocular dominance slabs in layer IV, thereby contacting layer III neurons that also receive input from the other eye. If one assumes the same degree of lateral spread for the axons of ON and OFF cells in layer IV within layer III, then corresponding ocular dominance gradients will be set up for each sign.

However, in comparing the two dimensions over which spatial gradients are generated, one difference becomes clear. In monkey and cat, all neurons encountered within a vertical electrode penetration have similar ocular dominance preferences (Hubel and Wiesel, 1968), presumably due to the strong radial connections between layers and the apical dendrites that span them. Since groups of neurons that have different targets are segregated into different layers (Gilbert and Kelly, 1975; Lund et al., 1975), with this arrangement, each group is assured of having a complete range of ocular dominance preferences. The laminar arrangement of ocular dominance we propose for the tree shrew raises the possibility that groups of projection neurons could differ in ocular dominance preferences. For example, there is some evidence that the neurons in layers II and IIIa project to a different region of extrastriate cortex from those in layer IIIc (Sesma and Meyer, 1989). Thus, in the tree shrew, ocular dominance could play an important role in the functional specialization of extrastriate visual areas. 


\section{Laminar stratification within layer $I V$}

A major insight into the functional significance of the sublaminar organization of layer IV in the tree shrew was the discovery by Harting et al. (1973) that layers IVa and IVb receive projections from different layers of the LGN. At the same time, Hubel and Wiesel (1972) discovered that neurons in the magnoand parvocellular layers of the monkey LGN project to separate strata within layer IVC. With this information, the significance of laminar stratification in layer IV seemed clear: to maintain the segregation of information supplied by different layers of the LGN. The results of the present study emphasize that laminar stratification within layer IV goes beyond that which would be predicted on the basis of LGN projections alone. We have been able to identify six separate strata within layer IV that differ in their pattern of projections to the supragranular layers. Studies of the intrinsic connections in the macaque striate cortex also suggest that laminar stratification within IVC is far more complex than a simple division into magno- and parvo-recipient sublaminae would indicate. Indeed, both IVC $\alpha$ and IVC $\beta$ contain sublaminae with different types of neurons and different patterns of connections (Fitzpatrick et al., 1985, 1987; Lund, 1987, 1990). Thus, it would appear that in both the monkey and the tree shrew, laminar stratification within layer IV plays an important rolc in restructuring the information supplied by the LGN, and transmitting this information to other cortical layers.

\section{References}

Adams JC (1981) Heavy metal intensification of DAB-based HRP reaction product. J Histochem Cytochem 29:775.

Blasdel GG, Fitzpatrick D (1984) Physiological organization of layer 4 in macaque striate cortex. J Neurosci 4:880-895.

Bullier J, Henry GH (1979) Laminar distribution of first-order neurons and afferent terminals in cat striate cortex. J Neurophysiol 42:12711281.

Bullier J, Henry GH (1980) Ordinal position and afferent input of neurons in monkey striate cortex. J Comp Neurol 193:913-935.

Callaway EM, Katz LC (1992) Development of axonal arbors of layer 4 spiny neurons in cat striate cortex. J Neurosci. 12:570-582.

Casagrande VA, Harting JK (1975) Transneuronal transport of tritiated fucose and proline in the visual pathways of tree shrew Tupaia glis. Brain Res 96:367-372.

Conley M, Fitzpatrick D, Diamond IT (1984) The laminar organization of the lateral geniculate body and the striate cortex in the tree shrew (Tupaia glis). J Neurosci 4:171-197.

Conway JL, Schiller PH (1983) Laminar organization of tree shrew dorsal lateral geniculate nucleus. J Neurophysiol 50:1330-1342.

Fitzpatrick D, Raczkowski D (1990) Innervation patterns of single physiologically identified geniculocortical axons in the striate cortex of the tree shrew. Proc Natl Acad Sci USA 87:449-453.

Fitzpatrick D, Itoh K, Diamond IT (1983) The laminar organization of the lateral geniculate body and the striate cortex in the squirrel monkey (Saimiri sciureus). J Neurosci 3:673-702.

Fitzpatrick D, Lund JS, Schmechel DE, Towles AC (1977) Distribution of GABAergic neurons and axon terminals in the macaque striate cortex. J Comp Neurol 264:73-91.

Fitzpatrick D, Lund JS, Blasdel GG (1985) Intrinsic connections of macaque striate cortex: afferent and efferent connections of lamina 4C. J Neurosci 5:3329-3349.

Geisert EE, Guillery RW (1979) The horizontal organization of stellate cell dendrites in layer IV of the visual cortex of tree shrews. Neuroscience 4:889-896.

Gilbert CD, Kelly JP (1975) The projections of cells in different layers of the cat's visual cortex. J Comp Neurol 163:81-106.

Gilbert CD, Wiesel TN (1979) Morphology and intracortical projections of functionally characterized neurones in the cat visual cortex. Nature 280:120-125

Harting JK, Diamond IT, Hall WC (1973) Anterograde degeneration study of the cortical projections of the lateral geniculate and pulvinar nuclei in the tree shrew (Tupaia glis). J Comp Neurol 150:393-440.

Hubel DH (1975) An autoradiographic study of the retino-cortical projections in the tree shrew (Tupaia glis). Brain Res 96:41-50.

Hubel DH, Wiesel TN (1961) Integrative action in the cat's lateral geniculate body. J Physiol (Lond) 155:385-398.

Hubel DH, Wiesel TN (1962) Receptive fields, binocular interaction and functional architecture in the cat's visual cortex. J Physiol (Lond) 160:106-154.

Hubcl DH, Wicscl TN (1968) Receptive fields and functional architecture of monkey striate cortex. J Physiol (Lond) 195:215-243.

Hubel DH, Wiesel TN (1972) Laminar and columnar distribution of geniculo-cortical fibers in the macaque monkey. J Comp Neurol 146: $421-450$.

Hubel DH, Wiesel TN (1977) Functional architecture of macaque monkey visual cortex. Proc R Soc Lond [Biol] 198:1-59.

Humphrey AL, Albano JE, Norton TT (1977) Organization of ocular dominance in tree shrew striate cortex. Brain Res 134:225-236.

Kretz R, Rager G, Norton TT (1986) Laminar organization of ON and OFF regions and ocular dominance in the tree shrew (Tupaia belangeri). J Comp Neurol 251:135-145.

Kuffler SW (1953) Discharge patterns and functional organization of mammalian retina. J Neurophysiol 16:37-68.

LeVay S, Gilbert CD (1976) Laminar patterns of geniculocortical projections in the cat. Brain Res 113:1-19.

LeVay S, McConnell SK, Luskin MB (1987) Functional organization of primary visual cortex in the mink (Mustela vison), and a comparison with the cat. J Comp Neurol 257:422-441.

Livingstone M, Hubel D (1984) Anatomy and physiology of a color system in the primate visual cortex. J Neurosci 4:309-356.

Lund JS (1973) Organization of neurons in the visual cortex, area 17, of the monkey (Macaca mulatta). J Comp Neurol 147:455-496.

Lund JS (1987) Local circuit neurons of macaque monkey striate cortex. I. Neurons of laminae $4 \mathrm{C}$ and 5A. J Comp Neurol 257:6092.

Lund JS (1990) Excitatory and inhibitory circuitry and laminar mapping strategies in the primary visual cortex of the monkey. In: Signal and sense. Local and global order in perceptual maps (Edelman GM, Gall WE, Cowan WM, eds), pp 51-66. New York: Wiley.

Lund JS, Lund RD, Hendrickson AE, Bunt AH, Fuchs AF (1975) The origin of efferent pathways from the primary visual cortex, area 17 , of the macaque monkey as shown by retrograde transport of horseradish peroxidase. J Comp Neurol 164:287-304.

Lund JS, Fitzpatrick D, Humphrey AL (1985) The striate cortex of the tree shrew. In: Cerebral cortex, Vol 3, Visual cortex (Peters A, Jones EG, eds), pp 157-205. New York: Plenum.

Martin KAC, Whitteridge D (1984) Form, function and intracortical projections of spiny neurones in the striate visual cortex of the cat. $\mathrm{J}$ Physiol (Lond) 353:463-504.

Muly E, Fitzpatrick D (1991) The morphological basis for binocular and ON/OFF convergence in tree shrew striate cortex. Soc Neurosci Abstr 17:844.

Muly E, Fitzpatrick D, Raczkowski D (1989) Efferent projections of layer 4 in tree shrew striate cortex: evidence for parallel pathways to the superficial layers. Soc Neurosci Abstr 15:1398.

Norton TT, Rager G, Kretz R (1985) ON and OFF regions in layer IV of striate cortex. Brain Res 327:319-323.

Ohzawa I, Freeman RD (1986a) The binocular organization of simple cells in the cat's visual cortex. J Neurophysiol 56:221-242.

Ohzawa I, Freeman RD (1986b) The binocular organization of complex cells in the cat's visual cortex. J Neurophysiol 56:243-259.

Palmer LA, Davis TL (1981) Receptive-field structure in cat striate cortex. J Neurophysiol 46:260-276.

Raczkowski D, Fitzpatrick D (1990) Terminal arbors of individual, physiologically identified geniculocortical axons in the tree shrew's striate cortex. J Comp Neurol 302:500-514.

Schiller PH (1982) Central connections of the retinal ON and OFF pathways. Nature 297:580-583.

Schiller PH, Finlay BL, Volman SF (1976) Quantitative studies of single-cell properties in monkey striate cortex. I. Spatiotemporal organization of receptive fields. J Neurophysiol 39:1288-1319.

Sesma MA, Meyer P (1989) Morphological features of area 17 efferents to extrastriate cortex in the tree shrew (Tupaia belangeri). Soc Neurosci Abstr 15:1399.

Shatz CJ, Stryker MP (1978) Ocular dominance in layer IV of the cat's 
visual cortex and the effects of monocular deprivation. J Physiol (Lond) 281:267-283.

Sherk H, Horton JC (1984) Receptive-field properties of cat's area 17 in the absence of on-center geniculate input. J Neurosci 4:381-393.

Skottun BC, Freeman RD (1984) Stimulus specificity of binocular cells in the cat's visual cortex: ocular dominance and the matching of left and right eyes. Exp Brain Res 56:206-216.
Tanaka K (1983) Cross-correlation analysis of geniculostriate neuronal relationships in cats. J Neurophysiol 49:1303-1318.

Usrey WM, Muly EC, Fitzpatrick D (1992) Lateral geniculate projections to the superficial layers of visual cortex in the tree shrew. J Comp Neurol, in press.

Zahs KR, Stryker MP (1988) Segregation of ON and OFF afferents to ferret visual cortex. J Neurophysiol 59:1410-1429. 